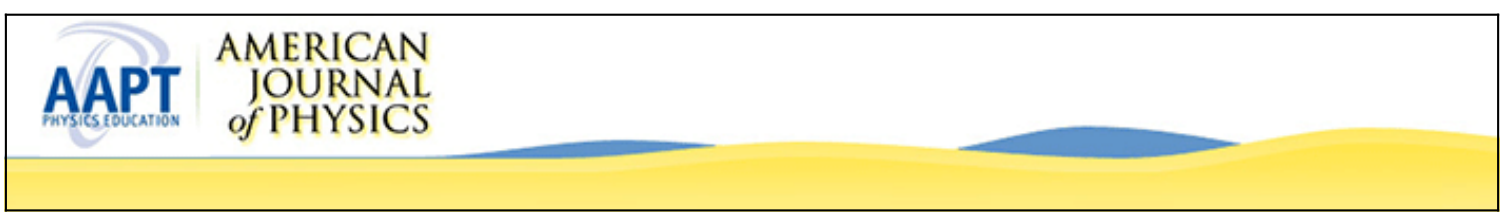

\title{
Microwave Faraday rotation
}

Kam L. Yan and W. P. Lonc

Citation: American Journal of Physics 43, 718 (1975); doi: 10.1119/1.9720

View online: http://dx.doi.org/10.1119/1.9720

View Table of Contents: http://scitation.aip.org/content/aapt/journal/ajp/43/8?ver=pdfcov

Published by the American Association of Physics Teachers

\section{Articles you may be interested in}

Faraday rotation in magnetically biased graphene at microwave frequencies

Appl. Phys. Lett. 102, 191901 (2013); 10.1063/1.4804437

Microwave Faraday Rotation in Nickel-Powder Artificial Dielectric

J. Appl. Phys. 38, 884 (1967); 10.1063/1.1709432

Method of Detecting Small Angles of Microwave Faraday Rotation

Rev. Sci. Instrum. 37, 1620 (1966); 10.1063/1.1720068

Microwave Faraday Rotation: Design and Analysis of a Bimodal Cavity

J. Appl. Phys. 29, 1692 (1958); 10.1063/1.1723027

A Method for Measuring Small Angles of Microwave Faraday Rotation

Rev. Sci. Instrum. 25, 394 (1954); 10.1063/1.1771079

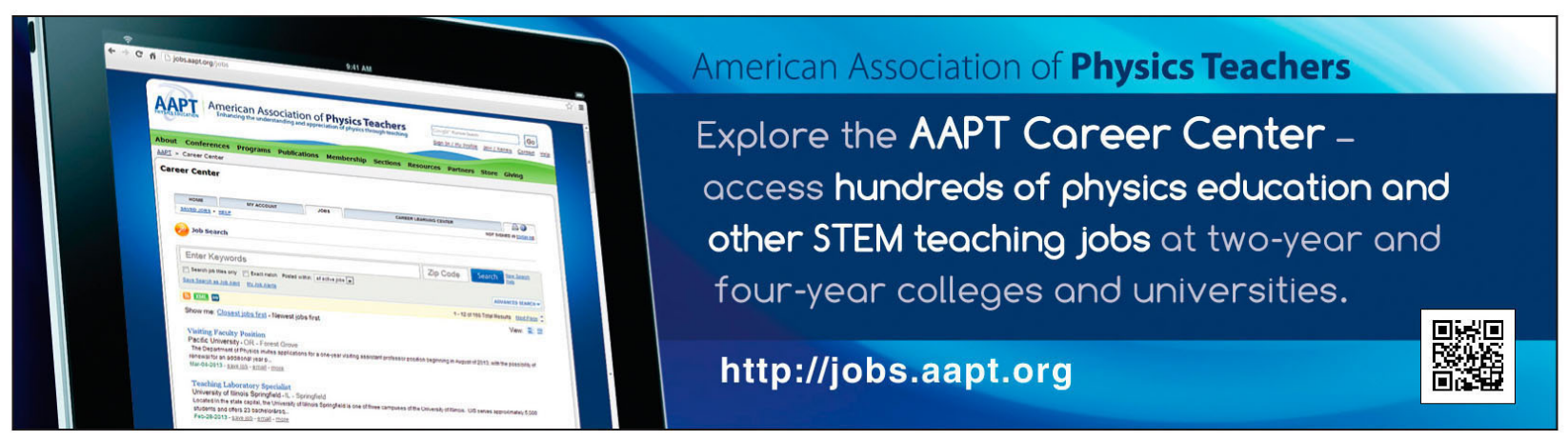


Kam L. Yan

W. P. Lonc, S. J.

Saint Mary's University

Halifax, Canada B3H 3 C3

(Received 21 June 1974; revised 10 September 1974)

By utilizing only commonly available microwave components and instruments, construction and performance details are given for a system capable of demonstrating the Faraday rotation effect at microwave frequencies with satisfactory quantitative results.

\section{INTRODUCTION}

The Faraday effect ${ }^{1}$ is a well-known effect both in the optical and radio-frequency parts of the spectrum, and it is of interest in fields such as radio astronomy in obtaining information on the existence and distribution of magnetic fields in space.

A study of the Faraday effect can be the subject of a 3-h undergraduate experiment. It is assumed that the laboratory has the basic $3-\mathrm{cm}$ (X-band) microwave components such as a klystron (or an equivalent) microwave source, an attenuator, and a microwave crystal detector. The only special item that might have to be acquired is the Faraday rotator itself.

The objectives of the experiment are to verify the linear dependence between the angle by which the plane of polarization is rotated and the strength of the applied magnetic field and to verify the dependence of the sense of angular rotation on the sense of the applied magnetic field. The experiment yields expected results for angles of rotation up to at least $70^{\circ}$.

\section{THEORY}

With reference to the objectives of this experiment, we choose to present a phenomenological discussion of the Faraday effect, in which we show that propagation of a wave in a magnetized ferrite does in fact yield a set of wave equations which can be interpreted in terms of a rotated plane of polarization and that the relationship between the magnetizing field and the rotation angle is linear for the magnitudes under consideration.

Consider the propagation of a linearly polarized wave of the magnetic field $H$ along the $z$ axis of a Cartesian coordinate system. Recall that a linearly polarized wave may be represented by the superposition of two oppositely rotating, circularly polarized waves of equal magnitude. If the axes are chosen such that the two circularly polarized waves reach maxima in the $y$ direction simultaneously at $z$ $=0$, it may be seen that these two circularly polarized waves of the magnetic field $\mathrm{H}$ can be written as

$$
H_{\mathrm{cw}}(z)=\cos [k(\mu) z]+j \sin [k(\mu) z]
$$

and

$$
H_{\text {ccw }}(z)=\cos [k(\mu) z]-j \sin [k(\mu) z]
$$

where $H_{\text {cw }}(z)$ represents the clockwise wave and $H_{\text {ccw }}(z)$ represents the counterclockwise wave and where $k(\mu)$ represents a functional dependence of the wave number $k$ on the permeability $\mu$. It can be readily shown that the plane of polarization does not change along $z$ as long as $\mu$ is the same for both circularly polarized waves.

Now, if we consider the effect of a magnetized ferrite to be expressed in terms of two permeabilities, $\mu_{1}$ and $\mu_{2}$, and if we associate $\mu_{1}$ with one of the circularly polarized waves and $\mu_{2}$ with the other, and if these two permeabilities differ in magnitude, then it can also be shown that the plane of polarization of the resultant plane wave has indeed rotated for increasing values of $z$. The sense of rotation will evidently depend on the initial assignment of the two permeabilities, whereas the angle of rotation will evidently depend on their relative magnitudes. Since the length of the Faraday rotator is a fixed quantity in this experiment, it is more appropriate to discuss the theory in terms of "angle of rotation" rather than the more usual "angle of rotation per unit length."

For example, the plane of polarization of the wave rotates by $\pi / 2$ radians in a distance of travel given by $z$ $=\pi /\left(k_{1}-k_{2}\right)$, where $k_{1}$ and $k_{2}$ are functions of $\mu_{1}$ and $\mu_{2}$, respectively. This relationship suggests that for arbitrary values of $z$ the direction of polarization of the wave has rotated by an angle $\theta$, given by

$$
\theta=\frac{1}{2} z\left(k_{1}-k_{2}\right)
$$

Consequently, the two rectangular components of the resultant plane wave $H_{r}(z)$ will be

$$
H_{y}=\cos \left[k_{1}\left(\mu_{1}\right) z\right]+\cos \left[k_{2}\left(\mu_{2}\right) z\right] \equiv H_{r}(z) \cos \theta
$$

and

$$
H_{x}=\sin \left[k_{1}\left(\mu_{1}\right) z\right]-\sin \left[k_{2}\left(\mu_{2}\right) z\right] \equiv H_{r}(z) \sin \theta,
$$

where $\theta$ is the angle by which $H_{r}(z)$ has rotated away from the $y z$ plane. It is fairly easy to show that, if one of the permeabilities (say, $\mu_{1}$ ) is a function of the magnetization $M$ within the ferrite and is given by

$$
\mu_{1}=1+\frac{4 \pi M}{H_{0}+\omega / \gamma}
$$

where $H_{0}$ is the applied magnetic field, $\omega$ is the angular frequency of the applied microwave radiation, and $\gamma$ 
$=\mu_{0} \epsilon / m$, and if the relationship between the wave number $k_{1}$ and the permeability $\mu_{1}$ is given by

$$
k_{1}^{2}=\left(\omega^{2} \epsilon / c^{2}\right) \mu_{1}
$$

and if it is assumed that $\omega>>H_{0}$, then application of binomial expansion yields the expression

$$
\theta \propto M .
$$

Then, assuming that the ferrite is operated in its linear magnetization characteristics so that $M$ is a linear function of $H_{0}$, we have a linear relationship between the angle of rotation and the applied magnetic field:

$$
\theta \propto H_{0}
$$

Finally, assuming $H_{0}$ to be a linear function of the current $I$ in the solenoid, we have the experimentally verifiable expression

$$
\theta \propto I
$$

where the vector properties of both $\theta$ and $I$ could be invoked to predict a change in the direction of $\theta$ for a change in direction of $\boldsymbol{H}_{\mathbf{0}}$.

\section{EXPERIMENTAL PROCEDURE}

The Faraday rotator, ${ }^{2}$ as supplied by the manufacturer, has rectangular waveguide input and output flanges. Both input and output ports are rectangular-to-circular waveguide transitions providing the necessary matching to the circular waveguide. The circular waveguide is designed to carry only the dominant transverse-electric $\left(\mathrm{TE}_{11}\right)$ mode. A ferrite core is located coaxially with the axis of the circular waveguide. The solenoid winding round the circular waveguide is connected to a variable dc power supply to obtain variable intensities of magnetic field. The ferrite core, in a given magnetized state, will rotate the plane of polarization by some amount that depends on the magnetic properties of the ferrite and the magnetic field strength of the solenoid. The direction of rotation is determined by the polarity of the applied magnetic field. The output of the Faraday rotator is analyzed by a suitable rotation of the rectangular waveguide existing between the Faraday rotator and the receiver (crystal detector).

Since one objective of this experiment is to verify the linear relationship between solenoid current and the corresponding angular change in the plane of polarization, an essential feature of the apparatus is a device for angle measurement. A simple and convenient device is a protractor set up in such a way that the receiver's axis of rotation coincides with the protractor's center. A pointer mounted on the receiver will then give fairly accurate angular readings. The experiment is here described in two slightly different configurations: (i) without any attenuator between klystron and Faraday rotator and (ii) with an attenuator. The attenuator constitutes a relatively large and constant load, whereas the Faraday rotator constitutes a relatively moderate but variable load. Hence, the klystron

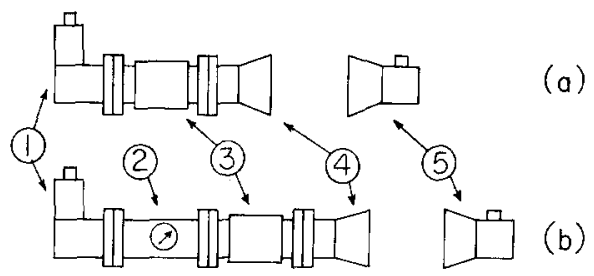

Fig. 1. Arrangement of the waveguide components. In (a), there is no attenuator between the klystron and Faraday rotator, whereas in (b) the attenuator is present. Components: 1, klystron; 2, attenuator; 3, Faraday rotator; 4 , transmitting horn; 5 , receiver assembly (including crystal detector). The protractor and pointer are not shown.

"sees" less variability in its load when driving both the attenuator and rotator rather than when driving the rotator alone, and this results in a more constant wave amplitude from the klystron.

For the first configuration, shown in Fig. 1(a), the Faraday rotator lies coaxially with the rotatable receiver, with the protractor and pointer suitably in place. A direct current is supplied to the solenoid and measured with a milliammeter. The angular reading corresponding to zero current in the solenoid and maximum response in the receiver is taken as zero; then, a nonzero current in the solenoid will give a nonzero angular measurement on the protractor after the receiver has been rotated for maximum response.

For the second configuration, shown in Fig. 1(b), the same procedures are followed, with the axes of the added attenuator, Faraday rotator, and the rotatable receiver all lying along a line.

In both configurations, the sense of the direction of rotation depends on the direction of the magnetic field and, hence, on the sense of the solenoid current. This effect may be verified by reversing the polarity of the solenoid current.

\section{RESULTS}

The data obtained from this experiment can be used to plot a graph showing rotation (in degrees) as a function of solenoid current, as in Fig. 2. From the set of data points from the second configuration (viz., with attenuation) a

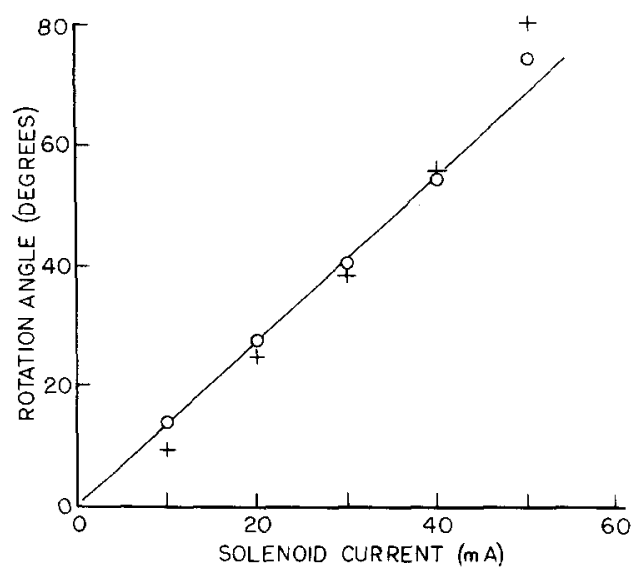

Fig. 2. Rotation of polarization as a function of solenoid current. The open circles $(0)$ are data points for the configuration that includes an attenuator between the klystron and rotator; the plus signs (+) are data points for the configuration in which the klystron connects directly to the rotator 
best-fit line can be drawn passing through most of these points. Data points from the first configuration (i.e., without attenuator) are slightly off this best-fit line. The significance of this graph is its verification of the linear relation between the solenoid current and the rotation of polarization. By reversing the polarity of the solenoid current, it is also shown that for a given magnitude of solenoid current the corresponding angular rotations are unchanged in magnitude; however, these angles of rotation are now in the opposite direction, which thus verifies the theoretical prediction.

\section{CONCLUSION}

In this experiment, it has been shown that the Faraday effect at microwave frequencies can be verified experimentally with relatively simple microwave equipment. The verification is within $10 \%$ for rotation of the plane of polarization from $0^{\circ}$ to $70^{\circ}$.
Note added in proof. In some instances, it may be much more convenient first to set the receiver at some angle and then to "tune" the current for maximum receiver output. This alternate procedure would avoid the possibility of random reflections interfering with the measurement procedure.

IJ. Smit and H. P. J. Wijn, Ferrites (Philips Technical Library, 1959), p. 97; F. Brailsford, Physical Principles of Magnetism (Van Nostrand, New York, 1966), p. 245; P. A. Matthews and I. M. Stephenson, Microwave Components (Chapman and Hall, London, 1968), p. 98.

${ }^{2}$ Lectronic Model 525, costing about \$35: Lectronic Research Laboratory, 715 Arch St., Philadelphia, PA 19106. The ferrite rod is approximately $6.2 \mathrm{~mm}$ in diameter and $10.2 \mathrm{~cm}$ long (the rod is of uniform diameter for approximately $8 \mathrm{~cm}$ of its length; then it tapers to $1 \mathrm{~mm}$ ) and of unspecified composition. The solenoid, which comes as part of the complete rotator, produces a field of approximately $25 \mathrm{At} / \mathrm{cm}(\mathrm{G})$ for a current of $60 \mathrm{~mA}$.

\section{PAPERWORK}

The first plutonium [ for nuclear weapons] was obtained by cyclotron bombardment and, of course, it was very, very scarce. Unweighable amounts came out. In St. Louis, the Washington University cyclotron was used for nothing else for two years or more; they kept it running all the time to get enough for the chemists to work out all the separation processes. I remember the first day when some plutonium that was really weighable came from the first plutonium producing plant, which was using the output of the gas-cooled pile at Oak Ridge (this must have been about 1943). When it came, it was brought by courier; I think it was Sam Allison who said, "Well, you know they're making progress, because this is the first time the amount of plutonium weighs more than the official papers that describe it." 